\title{
Tooth-implant connection: A bibliographic review
}

\author{
Celso Hita-Carrillo ${ }^{1}$, Manuel Hernández-Aliaga ${ }^{2}$, Jose-Luis Calvo-Guirado ${ }^{3}$ \\ ${ }^{1}$ Postgraduate Master in Implant Dentistry, Faculty of Medicine and Dentistry, University of Murcia \\ ${ }^{2}$ Associate Lecturer of General Dentistry, Faculty of Medicine and Dentistry, University of Murcia \\ ${ }^{3}$ Senior Leturer of General Dentistry, Faculty of Medicine and Dentistry, University of Murcia
}

Correspondence:

C/. Carrascas, 6

30564 Lorqui (Murcia) Spain

celsohita79@hotmail.com

Received: $11 / 12 / 2008$

Accepted: $30 / 07 / 2009$

\author{
Hita-Carrillo C, Hernández-Aliaga M, Calvo-Guirado JL. Tooth-implant \\ connection: A bibliographic review. Med Oral Patol Oral Cir Bucal. 2010 \\ Mar 1;15 (2):e387-94. \\ http://www.medicinaoral.com/medoralfree01/v15i2/medoralv15i2p387.pdf
}

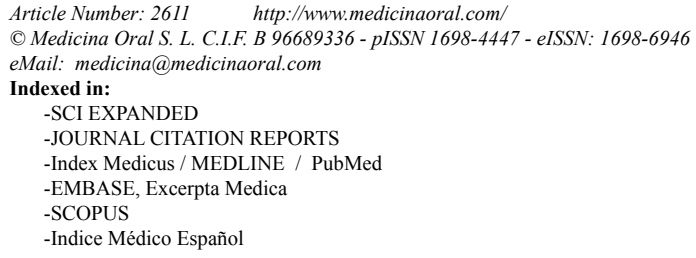

\begin{abstract}
The aim of this study was to carry out a bibliographic review of all available literature addressing the issue of whether or not the connection of teeth to implants by means of a prosthesis is a viable treatment alternative. Twenty articles from a variety of sources were analyzed and classified in order to draw conclusions. Articles were classified by type and an analysis was made of the different variables considered in each study, obtaining percentages of implant survival ranging from $84.4 \%$ to $100 \%$, prosthetic complications ranging from $80 \%$ to $90 \%$, and the incidence of dental intrusion ranging from 0 to $5.6 \%$. Biomechanical studies: Some articles studied models in order to assess different connections subjected to force, in which varying results were obtained. Rigid connections appeared to produce the most stress to the natural tooth, periodontal ligament and peri-implant bone; non-rigid connections reduced the stress to the bone, but increased stress to the prosthesis. Clinical studies: The results obtained were disparate. Studies in the medium or short-term show this as a viable treatment alternative, whereas some studies point to a greater risk of complications, although the use of rigid connection decreases the percentage of intrusion. Other bibliographic reviews have concluded that there is a need for more longitudinal studies on the viability of tooth-implant connection, also concluding that complications are greater when this is the chosen treatment. As a viable alternative with an acceptable success rate, this course of treatment is always associated with rigid connection rather than non-rigid connection. Although intrusion is avoided with rigid connection, this nevertheless remains inadvisable as the primary treatment choice.
\end{abstract}

Abstract

Key words: Tooth-implant connection, dental intrusion. 


\section{Introduction}

Tooth-implant connection by means of prosthetics remains a controversial issue due to the disparate results obtained in the various studies that have been conducted around the world. The differences in union between an osseointegrated implant and a natural tooth's union to the alveolar bone through periodontal ligament fibers mean that they behave very differently in response to the different masticatory forces, both natural and pathological. A number of published studies and articles have dealt with this issue in very different ways: bibliographic reviews $(1,2)$; in vivo studies $(3,4)$; in vitro biomechanical studies (5-7) and clinical case studies (8).

The aim of the present study was to conduct a bibliographic review of all the above literature published since the year 2000, to examine the connection of natural teeth to dental implants by means of tooth-to-implant supported prostheses and by doing so to determine if tooth-implant connection is a viable treatment option or not and, if so, in which cases might it be an appropriate option. This has involved the consideration of a range of related points and issues, which are as follows:

Only one study has been published that deals with complete-arch fixed partial dentures connecting teeth and implant abutments in patients with either normal or decreased periodontal support (9).

There is some controversy as to whether teeth should be extracted for the sake of avoiding tooth-implant connection. The risk of intrusion of natural teeth when combined with implants to support a fixed prosthesis means that the decision to extract is taken in order to avoid tooth-implant connections, even though natural teeth will survive implants ( $90 \%$ at the ten-year mark) (1).

Several studies have compared the frequency of biological complications with fixed prostheses fitted to implants or teeth or tooth/implant combinations over four to five years of functional life $(5,6)$.

The difference in movement of a tooth in good periodontal health and an osseointegrated implant can be five to twenty times greater. The use of non-rigid connectors is associated with dental intrusion, which occurs in $20 \%$ of cases of tooth-implant connection. A few studies reveal differences between rigid and non-rigid connections that are insignificant or only slightly significant $(5,6)$.

One study defends rigid connection on the grounds that it is a means of avoiding dental intrusion, rigidity not being at issue because it is thought that prostheses and implants possess the flexibility to accommodate differing mobility characteristics (10). Several other studies also defend rigid connection over non-rigid (2, 11-14).

\section{Materials and Methods}

Manual search methods were combined with Internet searches in various databases: Galilei um, Pubmed and
Cochrane Plus using "tooth-implant connected" and "tooth-implant connection" as key search phrases.

Science Direct was another database used through the University of Murcia General Library web-site at the Faculty of Medicine.

Seven further articles were obtained from other sources and the remaining texts, via the library website, were souced from on-line magazines and Biomedical E-journals.

As a result of these searches the review studied a total of twenty articles.

\section{Results}

The twenty articles selected for review were classified by type as follows: biomechanical studies $(40 \%)(5-7$, $10,15-18)$, clinical studies $(50 \%)(3,4,8,9,11-14,19,20)$ and bibliographic reviews of $(10 \%)(1,2)$. Of the articles reviewed we identified those that defend rigid connection $(50 \%)(2,3,5,6,8,11-14,16)$, and non-rigid connection $(10 \%)$, as well as the rest which do not mention it or do not differentiate it $(40 \%)(1,4,7,9,10,17-19)$ (Fig. 1).

It is also important to consider survival rates for implants connected to teeth and also the survival rate of the connecting prostheses, which were $84.4-100 \%$ for implants and $80-94.1 \%$ for prostheses $(1,2,4,8,9,11-14)$ (Fig. 2).

Other important data, included in some studies, are the percentages of dental intrusion associated to tooth-toimplant prosthetic connection, mainly with non-rigid connection whose values oscillate between $0-5.6 \%$ from study to study $(2,4,8,11-14)$ (Table 1).

In one study, Wang TM et al. 2004 (19) present a clinical case study in which it is proposed that intrusion of a natural tooth rehabilitated with fixed prosthesis, next to implant-supported rehabilitations could be a general problem of implant therapy, even when there is no tooth-implant connection involved. According to the study the problem has to do with bad adjustment of the tooth-supported prosthesis's inter-proximal and occlusal contact points with the adjacent implant-supported prosthesis. If good interproximal and occlusal contact is achieved, intrusion will be reversed. This poses the question of whether the interproximal, occlusal and lateral forces (and their proper adjustment in relation to the prosthesis) are more important than the implant-tooth connection or connection type (rigid or non-rigid).

Overall, the disparity between studies together with the differences in variables considered make comparison and analysis difficult. Nevertheless, the most significant data obtained from the clinical studies and bibliographic reviews indicate that implant survival rates oscillate between $84.4-100 \%$, prosthetic survival rates between $80-94.1 \%$ and intrusion between $0-5.6 \%$. 


\section{MANUSCRIPTS RELATED TO TOOTH- IMPLANT UNION}

RIGID CONNECTION $\square$ NON RIGID CONNECTION $\quad$ NO DIFFERENCES

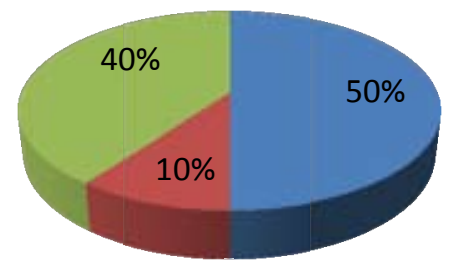

Fig. 1. Description of different manuscripts related to tooth-implant union.

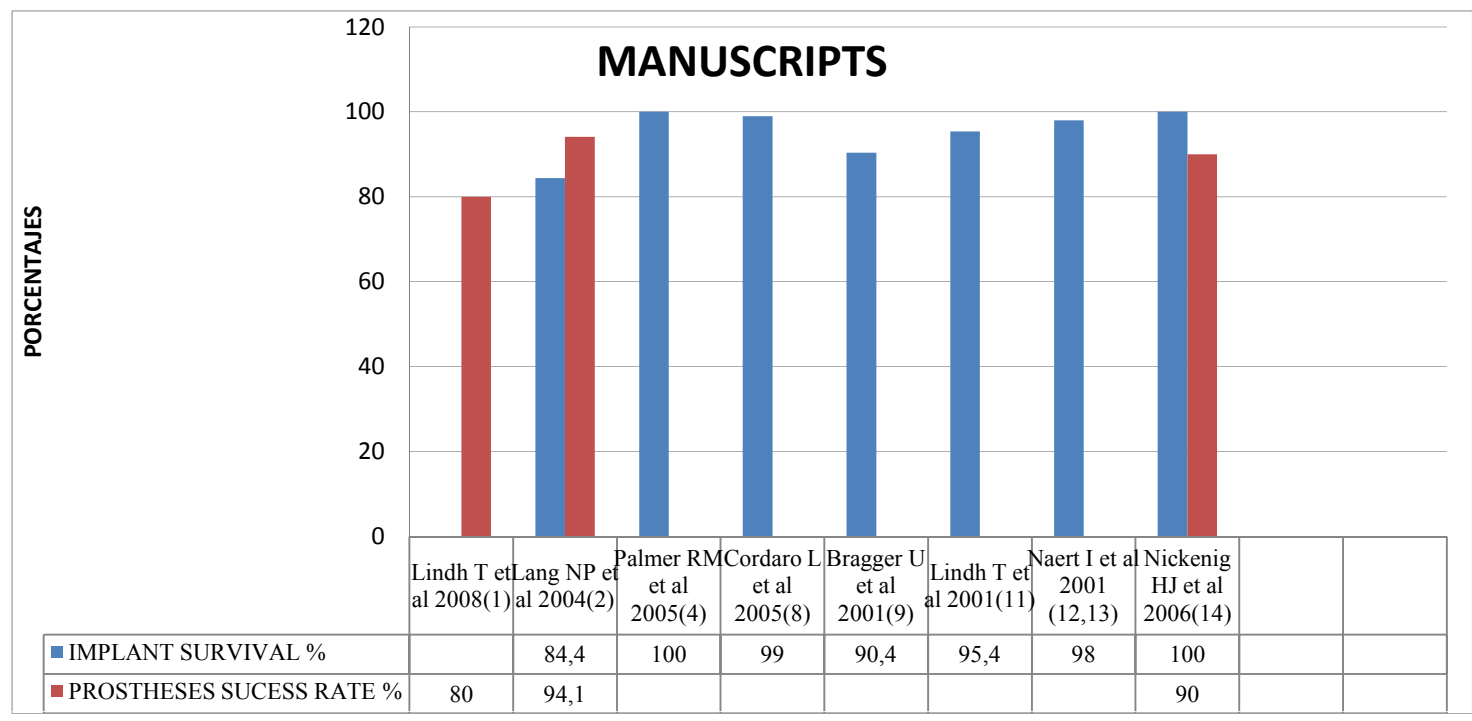

Fig. 2. Description of implant and prostheses success rate.

Table 1. Description of different manuscripts related of tooth intrusion.

\begin{tabular}{|c|c|c|}
\hline MANUSCRIPTS & $\begin{array}{c}\text { \% TOOTH } \\
\text { INTRUSION }\end{array}$ & $\begin{array}{c}\text { FOLLOW-UP } \\
\text { STUDY (years) }\end{array}$ \\
\hline Lang NP et al 2004 (2) & 5,2 & 5 \\
\hline Palmer RM et al 2005 (4) & 0 & 3 \\
\hline Cordaro L et al 2005 (8) & 5,6 & $2-7,8$ \\
\hline Lindh T et al 2001 (11) & 5 & 3 \\
\hline Naert I et al 2001 (12, 13) & 3,4 & $1,3-14,5$ \\
\hline Nickenig HJ et al 2006 (14) & 0 & $5-8$ \\
\hline
\end{tabular}




\section{Discussion}

In a study which researched the mechanical interactions of various systems of tooth-to-implant, rigid and nonrigid connections using non-linear $2 \mathrm{D}$ finite element analysis, Lin CL et al. 2006 (5) obtained stress values to the alveolar bone, the implant, and the prosthesis: stress was greater to the alveolar bone for teeth with periodontal problems, decreased slightly when implants were connected to two rather than one tooth and increased slightly when rigid connection was used; for stress to the implant, stress increased when there were periodontal problems, decreased slightly when one tooth connection became two and decreased with rigid connection; stress to the prosthesis did not vary according to periodontal health, nor did it vary significantly between connection to one or two teeth, but it did decrease a lot with the use of rigid connection. From this data it was concluded that tooth-to-implant connection can be a rational treatment choice in certain clinical situations, but that rigid connection should be used with caution, given that, although it decreases stress effects on bone, it increases tensions in implant and prosthesis particularly when there are periodontal problems. These are results that deserve some attention but, of course, there will always be a difference between research carried out on study models with all their limitations and the more complex and variable clinical reality.

Menicucci G et al. 2002 (10) studied some biomechanical aspects based on 2D and 3D finite element analysis. In two dimensions, a $50 \mathrm{~kg}$ load per piece was applied for 10 seconds, concentrated on the implant collar and tooth apex. In three dimensions, forces of $50 \mathrm{~kg}$ were applied for 5 milliseconds, distributed over the whole implant surface, although more to the implant collar and the the cervical area of the tooth's alveolar bone. It was concluded that a prolonged static load endangers periimplant bone more than alveolar bone so that it would appear that periodontal ligament plays a key role in stress distribution in tooth-to-implant connection.

Özçelic TB et al. 2007 (15) carried out an in vitro study of fixed tooth-to-implant supported prosthesis design using two different methods of stress analysis (2D and photoelastic). Forces of $250 \mathrm{~N}$ were applied to three models of fixed prosthesis with different types of connection, with results that showed greater stress to bone surrounding implants with rigid connection whilst the use of non-rigid connection reduced stress values. On these grounds the study defended non-rigid connection over rigid.

Ochiai KT et at 2003 (7) made a photoelastic stress analysis of implant-tooth connected prostheses with segmented and non-segmented abutments, obtaining similar stress values after loading the restoration, tooth and implant for each of the abutments. Loading the implant-supported zone showed a low transference of stress to the tooth and, when forces were not vertical, stress transference was slightly greater for non-segmented abutments. In spite of the study's limitations, we can deduce that stress distribution is similar whatever the abutment type, although this remains a simulated model rather than clinical reality.

Lin CL et al. 2006 (6) carried out a numerical simulation of the biomechanical interactions of tooth-implantsupported systems under different occlusal forces with both rigid and non-rigid connections. According to their results, lateral occlusal forces increase stress values significantly when compared with values for axial forces, independently of whether the connection was rigid or non-rigid; if occlusal force applied to a bridge pontic decreases, stress to the bridge's contact points decreases significantly. Stress values to prostheses were doubled for non-rigid connections compared to rigid. The difference between natural tooth and implant could be compensated by the use of non-rigid connection, although the differences found were not significant. Results also showed that for lateral displacements, non-rigid connection does not reduce stress efficiently. According to this study, that different micro-movements of different parts of the implant help to compensate the movement differences between tooth and implant and moreover that both implant and bone possess flexibility are two factors to be taken into consideration. It was observed that stress was concentrated differently for rigid connection (the lower part of the rigid connector) and non-rigid (in the concave surface of the connectors male component). The magnitude of occlusal force and the position of the point of occlusal force are more important than connector design for the influence on mechanical response (stress values and distribution) of implant systems and of alveolar bone.

Lin CL et al. 2008 (16) studied the biomechanical interactions in partial fixed tooth-to-implant supported prostheses with variations in the number of splinted teeth and the type of connection used, by means of finite element analysis. They found that loading conditions were the main factor determining stress to the bone, prosthesis and implant regardless of connector type or the number of teeth splinted. Prosthetic stress was increased by as much as three or four times in non-rigid connections compared to rigid. When a tooth was added to support the tooth-to-implant prosthesis it became more resistant to lateral occlusal forces. Reducing occlusal load in the bridge pontic area reduced prosthesis stress values, a finding that leads to the conclusion that non-rigid connectors compensate for the differing mobility of teeth and implants, but with the risk of increasing prosthesis stress when subjected to axial occlusal forces.

Maezawa N et al. 2007 (17) studied the connection of canines to implants in mandibular fixed prostheses using finite element analysis of three study models with 
different connections to the prosthesis. In particular, they examined maximum intercuspidation, canine protection and group function stresses to different abutments, alveolar bone and periodontal ligament. They found that for one-piece structures periimplant stress was lower and, by using stress-breakers, stress to the canines reduced. For stress to implants, the values were similar, group function generating the least stress. They concluded that these are acceptable restorations for patients with partial tooth loss.

Dalkiz M et al. 2002 (18) studied the difference between connecting an implant placed at the free end of one, two or three dental abutments in order to evaluate the stress to the abutments and to the bone ridge. After analyzing copious data they reached the conclusion that a prosthesis connected to three teeth reduced stress to the bone. The study was carried out using human mandibles to which a series of forces were applied; this method could not accurately replicate clinical reality but demonstrated an already established premise: that the more abutments support a fixed prosthesis, less stress to the bone, whether this is for teeth, implants or both.

Palmer RM et al. 2005 (4) made a three-year clinical study of fixed bridges connecting Astra Tech ST implants to natural teeth in nineteen subjects with Class II Kennedy divided into two groups, carrying out radiographic monitoring at the start of treatment and then annually for three years. They found statistically significant increases in probe depth, both for natural teeth and implants from treatment start to the three year mark, but marginal bone levels remained stable (Implant: start $0.65 \pm 0.42$; year one $0.63 \pm 0.47$; year two $0.88 \pm 0.55$; year three $0.78 \pm 0.64$. Tooth: start $2.29 \pm 0.82$; year one $2.41 \pm 0.8$; year two $2.38 \pm 1.02$; year three $2.68 \pm 0.86$ ). There were no cases of dental intrusion. The limitations to this study, regarding the number of patients, implants and study duration, mean that the outcome of these functional restorations after three years cannot permit recommending this treatment as a first choice, although the clinical and individual characteristics of each case might lead the practitioner to consider this type of treatment option.

Akça K et al. 2006 (3) have studied the relation between bite forces and the reaction of bone margins in fixed tooth-implant-supported prostheses with rigid connection, finding statistically significant differences between occlusal forces supported by teeth and implants (greater for implants than natural teeth $353,6114,17 \mathrm{~N}$ and $275,4813 \mathrm{~N}$, respectively) and stable bone margin levels after twenty-four months, although there was greater mesial loss than distal for implants $(0.28 \pm 0.519$ $\mathrm{mm}$ compared with $0,097 \pm 0,518 \mathrm{~mm})$. Although this is a short study period (twenty-four months) for determining long-term stability, it does, however, obtain data concerning the biomechanical differences between tooth and implant, the greater functional overload that implants suffer with a consequent bone loss, which in this study was reflected on the mesial side of implants connected to teeth.

Cordaro L et al. 2005 (8) made retrospective evaluations of complete-arch fixed prostheses on teeth and implants in patients with normal or reduced periodontal support. Nineteen subjects were assessed (nine with reduced periodontal support and ten with normal support) making periodic evaluations between the twenty-four month mark and ninety-four months. One implant was lost (99\% success rate) three implants suffered bone ridge losses of more than $2 \mathrm{~mm}$, there was $5.6 \%$ incidence of dental intrusion (four out of seventy-two) but only $13 \%$ of total intrusion occurred in teeth with normal periodontal support and associated with rigid connection; there were no cases of intrusion amongst teeth with reduced periodontal support when rigid connection was used. From this data, a low percentage of dental intrusion can be associated with teeth in good periodontal health connected to implants with complete-arch fixed prosthesis; the percentage of intrusion was minimal (13\% of a total of $5.6 \%$ intrusion).

Nickenig HJ et al. 2006 (14) examined survival rates and complications arising for fixed implant-tooth-supported prostheses. They assessed eighty-three patients treated at different clinics: $37.1 \%$ were posterior and mandibular (second lower premolar); $85 \%$ of implants were Branemark/Straumann; a third of prostheses were cemented and $26 \%$ telescopic; $39.3 \%$ were of three pieces and approximately one third were of five pieces or more; a third used non-rigid connection. $10 \%$ of these implant-tooth-supported prostheses were subjected to some modification after five years and 13\% after eight years. Rigid connections caused fewer problems and so required fewer modifications to the prosthesis (3 out of 56 , amongst rigid connections and eight out of twentyeight amongst non-rigid). There were no significant differences arising from the choice of implant system used. Not a single implant was lost out of a total of a hundred and forty-two but three teeth were lost out of a hundred and thirty two due to periodontal problems. At five years, $8 \%$ of teeth needed some kind of treatment and less than $1 \%$ of implants showed complications. It was concluded that with rigid connection, success rates for tooth-implant supported and implant-supported prostheses are very similar.

Brägger U et al. 2001 (9) compared the frequency of biological and technical complications in partial fixed prostheses on teeth, implants and combinations of both during four to five years functional life. The study used the ITI implant system in eighty-eight patients divided into three groups: implant-implant (thirty-three patients with eighteen prostheses); tooth-tooth (forty patients with fifty-eight prostheses) and tooth-implant (fifteen 
patients with eighteen prostheses). One prosthesis was lost in each group, so tooth-implant being the smallest group, this group showed a failure rate that was roughly double that of the other groups. Biological complications occurred in $9.6 \%$ of the implants and in $1.8 \%$ of dental abutments. More statistically significant technical complications were found in implant-supported prostheses, mainly associated with cases of bruxism. When extension prostheses were used, there were also more complications (thirteen out thirty-five compared with nine out of eighty one). However, the rate of loss over four to five years was similar for the three groups. The state of general health of individual patients did not associate significantly with biological failures, but bruxism and prosthetic extensions were associated with higher levels of technical failure. These are clearly factors which endanger the survival of fixed prostheses, whether they be tooth-supported, implant-supported or mixed, a fact that has been shown by numerous studies over the years. It is interesting that there was no association found between a patient's state of health and biological complications, given that ten patients out of thirty-two with health problems suffered complications whilst nine out of fifty three suffering complications had no health problem.

Naert I et al. $2001(12,13)$ made a comparative clinical study of implant-supported prostheses and tooth-implant-supported two-part prostheses analyzing: complications, failures, connection type (rigid or non-rigid), dental intrusion and marginal bone loss. Two groups were studied: the first of 123 patients with 140 prostheses (connecting 339 implants to 313 teeth with an average age of 71.8 years (ranging between 20 and 79 years) monitored over between 1.5 and 15 years (average: 6.5 years); the second of 123 patients with 123 prostheses (329 implants were connected) with an average age of 52.3 years (ranging between 22 and 78 years) monitored for between 1.3 and 14.5 years (average: 6.2 years). Both groups showed good success rates for implants, 95\% (ten implants failed) and $98.5 \%$ (one failure) respectively. $3.5 \%$ periapical lesions were registered, $0.6 \%$ dental fracture, $1 \%$ of teeth had to be extracted, $3.4 \%$ dental intrusion and in three patients the structure broke. For the inter-connected implants group there were only two screw abutment fractures. Marginal bone loss for tooth-implant-supported prostheses throughout the study period was $0.7 \mathrm{~mm}$ greater than for implantsupported prostheses. There were no significant differences in bone loss between non-rigid tooth-implant and implant-implant prostheses, but there were significant differences in bone loss, bone loss being greater for tooth-implant prostheses than for implant-implant. The study concluded that the first treatment option should be implant-implant connection but if this is not possible then rigid connection should be used in order to avoid intrusion, although this will involve greater marginal bone loss. From all this data and conclusions it can be seen that connections between teeth and implants generate more problems both clinically and for the patient (whether this is for teeth periapical lesions, fractures, exodontia, intrusion) for prostheses (structural or porcelain fracture) or for the implant (screw abutment fracture, marginal bone loss) than when implants are connected to each other (when there might be complications for the implant or prosthesis but generally fewer). Lindh T et al. 2001 (11) made a study of prostheses connected to implants and teeth in which they evaluated the survival of implants and marginal bone loss. They collated data from 111 patients with 185 implants following a single protocol, finding $95.4 \%$ implant survival rate over three years. 6 out of 185 suffered osseointegration loss; 4 out of 183 developed some type of periimplantitis infection and $5 \%$ of cases suffered intrusion, associated with reconstructions with non-rigid connection: they conclude that rigid connection helps prevent dental intrusion.

Chee W et al. 2006 (20) made a clinical study of modifications to non-rigid connection. They designed a tooth-implant-supported fixed prosthesis with non-rigid connection between implant and pontic, comparing this with other studies assessing non-rigid connections between teeth and pontic, producing high levels of intrusion. According to this study rigid connection has adverse effects on implants in the long term, but non-rigid connection can intrude the tooth. One way of reducing intrusion and long term adverse effects on implants could be non-rigid connection with distal attachment to the pontic on implant-supported restorations.

Lang NP et al. 2004 (2) reviewed a total of thirteen studies by means of meta-analysis. The results of this review indicated that $90.1 \%$ of fixed tooth-implant-supported prostheses had survived after five years and $82.1 \%$ after ten years. The survival rate for fixed tooth-implantsupported prostheses at five years was $94.1 \%$ and $77.8 \%$ at ten years. In five years $3.2 \%$ of tooth abutments and $15.6 \%$ of implants were lost. Over five years there was a $5.2 \%$ incidence of dental intrusion, mainly in non-rigid connections. It was concluded that fixed prostheses supported by implants alone had a greater survival rate compared with tooth-implant-supported prostheses. However, for certain clinical situations, the authors also state that further longitudinal studies are needed before fixed prosthesis tooth-to-implant connection can be ruled out altogether. Lindh T, 2008 (1) poses the question of whether we should extract teeth in order avoid tooth-implant combinations. He has reviewed the available literature, finding that many authors have not found major risks with such combinations, although there are still more complications produced and worse prognosis for fixed prostheses connecting teeth and implants. He 
reviewed the survival rates for implant-supported prostheses, finding a 95.4/\% survival rate for implants and $95 \%$ for prostheses. He also compared survival at ten years of tooth-supported prostheses (89.2\%), implantsupported $(86.7 \%)$ and tooth-implant-supported $(80 \%)$. He points out that all the data used comes from studies with fairly different designs and this makes comparison difficult. In this way very disparate data emerged regarding which studies did or did not reveal significant differences in marginal bone response to prostheses supported by tooth and implant combinations. Other studies reviewed identified a risk of dental failure due to caries or periodontal and/or endodontic problems. As for dental intrusion, the studies revealed that rigid connection reduced intrusion considerably.

The overall disparity of the studies that we have reviewed here, together with their design and the variables affecting each of them, make comparison and interrelation very difficult.

Amongst the in vitro biomechanical studies we have found studies that conclude that tooth-implant connection is a viable treatment alternative, so long as nonrigid connection is used with caution as this increases stress on the prosthesis $(5-7,16)$. In others, however, it was found that non-rigid connection decreased stress to the bone, and that greater numbers of abutments also decrease stress to the bone $(15,18)$. There again, one study found no significant differences in stress distribution (7). We must not forget, however, that the limitations of these in vitro studies, in terms of model design (which simulate reality but cannot reproduce it), application, duration, and force distribution, cannot compare to clinical realities.

In clinical studies of patients, there are some studies that consider tooth-implant connection as viable, achieving functional restorations $(4,11)$. As for connection type, rigid connection is seen as the better option in order to avoid dental intrusion $(8,11-14)$, but one study did not identify significant differences in the long term (20). Regarding overall prosthetic and clinical complications, various studies pointed to higher levels with tooth-implant connections $(12,13)$ although these are sometimes associated with cases of bruxism and prosthetic extension (9). However there are also those studies that failed to find significant differences compared with prostheses supported by implants alone. The disparity between the studies in terms of the number of patients involved, the length of study period and differences between clinical cases, make the comparison of data difficult. Furthermore, the individual skills of the practitioner involved is a factor that was not considered in any of the studies and which is undoubtedly a very important one, affecting the long term success of any restoration.

From the bibliographic reviews included in the present study $(1,2)$ very similar results were obtained with re- gard to long term survival rates although these was greater for implant-supported restorations, which also showed lower rates of complication and better prognosis. They agree that more longitudinal studies are needed and that these should be of homogenous design in order to facilitate comparison.

\section{Conclusions}

From all the literature reviewed we have reached the following conclusions:

1. The studies all have their limitations with regard to: the reproduction of clinical reality when in vitro models are used, the forces applied and the response of models to these forces; sample sizes; duration of study periods; disparity of groups studied; variables taken into consideration in each study. All this makes it difficult to compare articles and to draw absolute conclusions.

2. Rigid connection achieves better outcomes with regard to avoiding dental intrusion, although it will produce greater marginal bone loss, with a corresponding increase in probe depth around the supporting abutment (tooth or implant).

3. Tooth-implant-supported prostheses suffer higher failure rates and complications with more trouble for both patient and clinician and less durability.

4. More longitudinal studies are needed of tooth-implant connected prostheses before this type of treatment can be recommended as a first choice. The connection of implant-implant remains the safest option although, depending on the particular clinical situation and other case characteristics as well as the individual patient, it can be a viable alternative with an acceptable success rate.

\section{References}

1. Lindh T. Should we extract teeth to avoid tooth-implant combinations?. J Oral Rehabil. 2008;35 Suppl 1:44-54.

2. Lang NP, Pjetursson BE, Tan K, Brägger U, Egger M, Zwahlen M. A systematic review of the survival and complication rates of fixed partial dentures (FPDs) after an observation period of at least 5 years. II. Combined tooth--implant-supported FPDs. Clin Oral Implants Res. 2004;15:643-53.

3. Akça K, Uysal S, Cehreli MC. Implant-tooth-supported fixed partial prostheses: correlations between in vivo occlusal bite forces and marginal bone reactions. Clin Oral Implants Res. 2006;17:331-6.

4. Palmer RM, Howe LC, Palmer PJ. A prospective 3-year study of fixed bridges linking Astra Tech ST implants to natural teeth. Clin Oral Implants Res. 2005;16:302-7.

5. Lin CL, Wang JC, Kuo YC. Numerical simulation on the biomechanical interactions of tooth/implant-supported system under various occlusal forces with rigid/non-rigid connections. J Biomech. 2006;39:453-63.

6. Lin CL, Chang SH, Wang JC, Chang WJ. Mechanical interactions of an implant/tooth-supported system under different periodontal supports and number of splinted teeth with rigid and non-rigid connections. J Dent. 2006;34:682-91.

7. Ochiai KT, Ozawa S, Caputo AA, Nishimura RD. Photoelastic stress analysis of implant-tooth connected prostheses with segmented and nonsegmented abutments. J Prosthet Dent. 2003;89:495-502. 8. Cordaro L, Ercoli C, Rossini C, Torsello F, Feng C. Retrospective 
evaluation of complete-arch fixed partial dentures connecting teeth and implant abutments in patients with normal and reduced periodontal support. J Prosthet Dent. 2005;94:313-20.

9. Brägger U, Aeschlimann S, Bürgin W, Hämmerle CH, Lang NP Biological and technical complications and failures with fixed partial dentures (FPD) on implants and teeth after four to five years of function. Clin Oral Implants Res. 2001;12:26-34.

10. Menicucci G, Mossolov A, Mozzati M, Lorenzetti M, Preti G. Tooth-implant connection: some biomechanical aspects based on finite element analyses. Clin Oral Implants Res. 2002;13:334-41.

11. Lindh T, Dahlgren S, Gunnarsson K, Josefsson T, Nilson H, Wilhelmsson P, et al, Tooth-implant supported fixed prostheses: a retrospective multicenter study. Int J Prosthodont. 2001;14:321-8.

12. Naert IE, Duyck JA, Hosny MM, Quirynen M, Van Steenberghe D. Freestanding and tooth-implant connected prostheses in the treatment of partially edentulous patients Part II: An up to 15-years radiographic evaluation. Clin Oral Implants Res. 2001;12:245-51.

13. Naert IE, Duyck JA, Hosny MM, Van Steenberghe D. Freestanding and tooth-implant connected prostheses in the treatment of partially edentulous patients. Part I: An up to 15 -years clinical evaluation. Clin Oral Implants Res. 2001;12:237-44.
14. Nickenig HJ, Schäfer C, Spiekermann H. Survival and complication rates of combined tooth-implant-supported fixed partial dentures. Clin Oral Implants Res. 2006;17:506-11.

15. Ozçelik T, Ersoy AE. An investigation of tooth/implant-supported fixed prosthesis designs with two different stress analysis methods: an in vitro study. J Prosthodont. 2007;16:107-16.

16. Lin CL, Wang JC, Chang WJ. Biomechanical interactions in tooth-implant-supported fixed partial dentures with variations in the number of splinted teeth and connector type: a finite element analysis. Clin Oral Implants Res. 2008;19:107-17.

17. Maezawa N, Shiota M, Kasugai S, Wakabayashi N. Three-dimensional stress analysis of tooth/lmplant-retained long-span fixed dentures. Int J Oral Maxillofac Implants. 2007;22:710-8.

18. Dalkiz M, Zor M, Aykul H, Toparli M, Aksoy S. The three-dimensional finite element analysis of fixed bridge restoration supported by the combination of teeth and osseointegrated implants. Implant Dent. 2002;11:293-300.

19. Wang TM, Lee MS, Kok SH, Lin LD. Intrusion and reversal of a free-standing natural tooth bounded by two implant-supported prostheses: a clinical report. J Prosthet Dent. 2004;92:418-22.

20. Chee W, Jivraj S. Connecting implants to teeth. Br Dent J. 2006;201:629-32. 